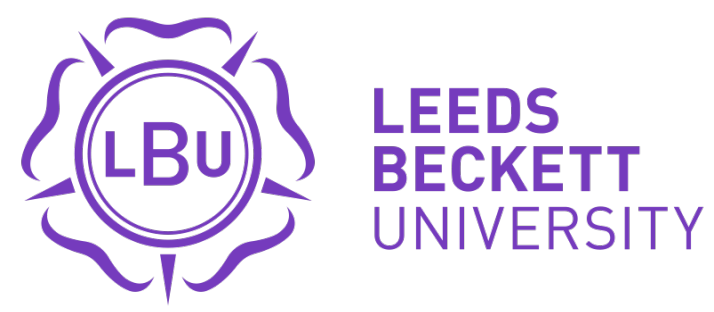

Citation:

Ntoumanis, N and Ng, JY and Barkoukis, V and Backhouse, SH (2014) Personal and psychosocial predictors of doping use in physical activity settings: a meta-analysis. Sports medicine (Auckland, N.Z.), 44 (11). 1603 - 1624. ISSN 0112-1642 DOI: https://doi.org/10.1007/s40279-014-0240-4

Link to Leeds Beckett Repository record:

https://eprints.leedsbeckett.ac.uk/id/eprint/61/

Document Version:

Article (Accepted Version)

The aim of the Leeds Beckett Repository is to provide open access to our research, as required by funder policies and permitted by publishers and copyright law.

The Leeds Beckett repository holds a wide range of publications, each of which has been checked for copyright and the relevant embargo period has been applied by the Research Services team.

We operate on a standard take-down policy. If you are the author or publisher of an output and you would like it removed from the repository, please contact us and we will investigate on a case-by-case basis.

Each thesis in the repository has been cleared where necessary by the author for third party copyright. If you would like a thesis to be removed from the repository or believe there is an issue with copyright, please contact us on openaccess@leedsbeckett.ac.uk and we will investigate on a case-by-case basis. 


\title{
Personal and Psychosocial Predictors of Doping Use in Physical Activity Settings: A
}

\section{Meta-Analysis}

\author{
Nikos Ntoumanis \\ Curtin University, Australia \\ Johan Y. Y. Ng \\ The Chinese University of Hong Kong \\ Vassilis Barkoukis \\ Aristotle University of Thessaloniki, Greece \\ Susan Backhouse \\ Leeds Metropolitan University, UK
}

Johan Y. Y. Ng: Department of Sports Science and Physical Education Faculty of Education, The Chinese University of Hong Kong Shatin, N.T., Hong Kong

Vassilis Barkoukis: Department of Physical Education and Sport Sciences, Aristotle University of Thessaloniki, 57001, Thermi, Thessaloniki, Greece

Susan Backhouse: Institute of Sport, Physical Activity and Leisure, Leeds Metropolitan University, Fairfax Hall 217, Headingley Campus, Leeds LS6 3QS, United Kingdom

Address correspondence: Nikos Ntoumanis, $\mathrm{PhD}$

Health Psychology \& Behavioural Medicine Research Group

School of Psychology \& Speech Pathology

Curtin University,

GPO Box U1987

Perth, Western Australia, 6845

Nikos.Ntoumanis@curtin.edu.au 


\title{
Key points
}

- A meta-analysis including 63 independent datasets was conducted.

- Use of legal supplements, perceived social norms, and positive attitudes towards doping were strong positive correlates of doping intentions and behaviors.

- Morality and self-efficacy to refrain from doping were negatively associated with doping intentions and behaviors.

\begin{abstract}
Background: There is a growing body of empirical evidence on demographic and psychosocial predictors of doping intentions and behaviors utilizing a variety of variables and conceptual models. However, to date there has been no attempt to quantitatively synthesize the available evidence and identify the strongest predictors of doping.

Objectives: Using meta-analysis, we aimed to (i) determine effect sizes of psychological (e.g., attitudes), social-contextual factors (e.g., social norms), and demographic (e.g., sex and age) variables on doping intentions and use, (ii) examine variables which moderate such effect sizes, and (iii) test a path analysis model, using the meta-analyzed effect sizes, based on variables from the Theory of Planned Behavior.
\end{abstract}

Data sources: Articles were identified from online databases, by contacting experts in the field, and searching the World Anti-Doping Agency website.

Study eligibility criteria and participants: Studies that measured doping behaviors and/or doping intentions, and at least one other demographic, psychological, or social-contextual variable were included. We identified 63 independent datasets.

Study appraisal and synthesis method: Study information was extracted by using predefined data fields and taking into account study quality indicators. A random effects meta-analysis was carried out, correcting for sampling and measurement error, and 
identifying moderator variables. Path analysis was conducted on a sub-set of studies that utilized the Theory of Planned Behavior.

Results: Use of legal supplements, perceived social norms, and positive attitudes towards doping were the strongest positive correlates of doping intentions and behaviors. In contrast, morality and self-efficacy to refrain from doping had the strongest negative association with doping intentions and behaviors. Furthermore, path analysis suggested that attitudes, perceived norms, and self-efficacy to refrain from doping predicted intentions to dope, and indirectly, doping behaviors.

Limitations: Various meta-analyzed effect sizes were based on a small number of studies, which were correlational in nature. This is a limitation of the extant literature.

Conclusions: This review identifies a number of important correlates of doping intention and behavior, many of which were measured via self-reports and were drawn from an extended theory of planned behavior framework. Future research might benefit from embracing other conceptual models of doping behavior and adopting experimental methodologies that will test some of the identified correlates in an effort to develop targeted anti-doping policies and programs. 


\section{Introduction}

According to the World Anti-Doping Agency [1], doping behavior refers to the use of illegal performance enhancing drugs and methods to improve performance. Anti-doping rules and regulations in sport are founded upon the premise that doping use violates the rules of competition and the spirit of sport, and it can lead to health risks in users [1]. Nonetheless, doping behaviors are not uncommon across all levels of sport and exercise participation $[2,3]$. In a study conducted in a gym setting, more than $10 \%$ of participants self-reported engaging in doping behaviors [4]. Amongst competitive bodybuilders, the figures are as high as $77.8 \%$ [5]. In competitive sport, the prevalence statistics are wide-ranging and appear to be dependent on the measurement technique employed. Questionnaire-based evidence indicates that approximately $10 \%-15 \%$ of elite and sub-elite athletes reported using doping substances in the past [6]. The use of indirect measures (e.g., Implicit Association Test or the Randomized Response Technique) indicates that the prevalence of doping use in competitive sport can be as high as 30\% [7-9]. The Athlete Biological Passport paradigm has also provided estimates of the prevalence of blood doping. Analysis of samples from 2737 international athletes provided a mean estimate of $14 \%$ for the combined sample, but subpopulation analysis highlighted wide ranging estimates from $1 \%$ to $48 \%$ [10]. All these figures are in stark contrast to the fact that, year on year, less than $2 \%$ of drug tests analyzed by WADA laboratories prove positive.

Therefore, despite the efforts of WADA, the International Olympic Committee (IOC) and global sports federations, drug use in sport and exercise settings is on the rise [11] with a recent high profile case being that of the cyclist Lance Armstrong. The reality is that detection techniques are not keeping pace with developments in the pharmaceutical industry [12] and the need for anti-doping education is greater than ever [11]. Thus, preventive and educational action must be afforded greater attention and resource to reduce instances of 
doping. Such preventative action necessitates an understanding of the psycho-social predictors of doping intentions and behavior. Research examining such predictors has significantly increased over the last decade. As such, a number of positive and negative correlates at the personal and socio-contextual level (e.g., achievement goal orientations, moral values, social norms) have been identified. In line with health-related behavior research, most studies in the doping field have adopted the theory of planned behavior (TPB) $[13,14]$ as a broader theoretical background to examine the psycho-social mechanisms that may lead to doping in sport. In this theory, behavior is viewed as a distal or proximal outcome of 1) intention, 2) attitude, 3) subjective norm, and 4) perceived behavioral control. Attitudes represent an individual's positive or negative evaluations of performing the behavior in question. Subjective norms are conceptualized as the pressure that individuals perceive from significant others to engage (or not) in the target behavior. Perceived behavioral control represents one's evaluation about their capabilities to perform the behavior. As such, it may reflect obstacles encountered in past behavioral performances. Attitudes, subjective norms and perceived behavioral control are proposed to influence behavior through their influence on intention to perform the behavior. Hence, intentions represent an individual's proximal predictor of doping behavior.

Researchers utilizing TPB have documented the ability of doping attitudes, perceived behavioral control and subjective norms to predict doping intention and self-reported doping behavior [e.g., 15-17]. These studies have involved a variety of populations including elite athletes [16], gym users [18], and adolescents [17, 19]; suggesting generalizability of findings across different samples and settings. Alongside the core TPB variables, researchers have also used constructs outside the TPB framework to predict doping intentions and behaviors. The extension of the TPB approach was undertaken because of a) the need to investigate morality issues bearing in mind that doping is considered an immoral behavior, b) recent 
developments in TPB research in other areas of enquiry (e.g. smoking), showing that the addition of new constructs (i.e., descriptive norms, situational temptation) improves the predictive ability of the TPB model, and c) recent arguments suggesting the use of integrative approaches $[14,20]$ to incorporate distal predictors of behavior (e.g., sportspersonship, moral disengagement, or achievement goals in the case of doping) and demographics. As an example of such new approaches, Lucidi et al. [17] measured participants' moral disengagement (i.e., disassociating oneself from the moral implications of one's unethical actions) and showed that this variable predicted doping intentions and behaviors. Barkoukis et al. [21] found that athletes with high sportspersonship (e.g., prosocial attitudes and behaviors), autonomous motivation (i.e., motivation resulting from enjoyment or personal value) and mastery-oriented achievement goals (i.e., emphasis on personal improvement and effort) reported lower doping intentions compared to those with low sportspersonship, controlled motivation (i.e., motivation resulting from pressure, social approval or feelings of guilt) and performance oriented achievement goals (i.e., emphasis on displaying normative superiority). Mixed evidence accompanies research exploring the demographic predictors of doping use. For instance, males, compared to females, have been found to be at a greater risk for doping across samples of adolescents [22] and competitive athletes [23, 24]. However, Wiefferink et al. [18] did not find any effects of sex or age on self-reported use of doping substances in a sample of gym and fitness users.

The aforementioned findings highlight that doping use is complex and is probably predicted by a variety of situational and personal variables. The vast majority of studies in this area are correlational in design and doping behavior is self-reported. Anti-doping interventions from a psycho-social perspective are scarce [25]. As an exception, Elliot et al. [26] designed an intervention consisting of eight 45-minute meetings held during sport team training in US high schools. This was a peer-led intervention which did not focus exclusively 
on doping; students engaged in cooperative learning exercises regarding healthy nutrition and strength training regimes, as well as the effects of legal and illegal supplements and other substances on performance. The intervention was sex-specific targeting different aspect of males' (i.e., steroid use; project Adolescents Training and Learning to Avoid Steroids ATLAS) and females' (i.e., disordered eating behavior; project Athletes Targeting Healthy Exercise and Nutrition Alternatives - ATHENA) behaviors. Using a randomized control trial design, Elliot et al. showed that the program significantly reduced the use of recreational drugs and doping substances in participants one to three years after the students graduated from high school. However, the effect sizes found were very small.

To date, only one systematic narrative literature review has been performed to synthesize the research findings in this area [25]. The authors reviewed 103 articles focusing on attitudes, values and beliefs towards doping, predictors and precipitating factors in antidoping education and prevention programs. Most included studies examined attitudes towards doping. Only 21 studies examined use of performance enhancing drugs and most of those studies examined risk factors for anabolic steroid use amongst weight lifters or body builders. As such, the findings from this review may not be generalizable to other populations and settings, and are better suited for understanding attitudes toward doping but not necessarily doping intention and use. By conducting a meta-analysis of existing studies, researchers can provide a vigorous test of theoretical frameworks utilized in the field of doping, by incorporating a large number of studies using different study designs and including participants with varying demographic backgrounds. A meta-analysis can accumulate, quantify, and summarize existing empirical evidence controlling for sampling and measurement errors which cannot be taken into account by narrative reviews. By quantifying the magnitude of the size of effect between variables and testing the homogeneity of this effect across different groups or contexts, researchers can identify key psycho-social 
predictors of doping intention and behavior, so that resources are specifically targeted on these risk factors. Further, based on the results of meta-analysis, researchers may refine existing theoretical frameworks or develop new ones that could better explain the links between demographic and psychosocial variables with doping intention and behavior.

\subsection{Objectives of the Current Study}

In order to provide a statistical synthesis of research findings in the literature, we conducted a meta-analysis of existing studies. The first aim of this study was to identify the strongest psycho-social correlates (both positive and negative) of doping intentions and use (behaviors). The studies reporting the results of the ATLAS/ATHENA interventions were included in our meta-analysis; although these studies did not focus exclusively on doping, doping behavior was one of their outcome measures and hence they met our inclusion criteria. In addition to psychological (e.g., attitudes) and social-contextual factors (e.g., social norms), the predictive effects of demographic variables (e.g., sex and age) on doping intentions and behaviors were also examined. Results from intervention studies were also meta-analyzed in order to examine the effectiveness of anti-doping interventions by comparing the findings between the experimental and control arms. By conducting a meta-analysis, one can examine whether real effects (i.e., effects that are unlikely to be due to chance) exist between two variables. Moreover, the size of such effects could be calculated using a standardized metric, which is useful for comparison purposes.

The second aim of this study was to identify moderator variables (i.e., whether the effect size associated with the relationship between two variables varies at different levels of the moderator variable). Testing for moderators is important because researchers can identify whether certain effects, such as the influence of social norms on doping intentions, need to be dealt with differently in various groups (e.g., males versus females) or in certain contexts (e.g., competitive athletes versus gym users). Further, moderator analysis could help 
researchers to explore whether doping interventions are equally effective across populations and settings. The third aim of this study was to test a path analysis model, using the metaanalyzed effect sizes, using variables proposed by TPB [13]. We chose TPB for the path analysis because most of the empirical studies in the extant literature were based on this theory.

\section{Methods}

\subsection{Information Sources and Search Strategy}

A literature search was conducted to identify studies that could be included in the meta-analysis. PsycINFO, PsycARTICLES, PubMed, Google Scholar, and Medline were searched using combinations of keywords specifying the subject (e.g., doping, doping intention, steroid) and the context of interest (e.g., sport) with no date limits. The full electronic search strategy is described in Electronic Supplementary Material Appendix S1. In addition, the WADA website was searched for reports of previous studies funded by the agency, and the reference lists of included articles were scanned for relevant manuscripts that were not retrieved from the database searches. To locate and retrieve information from unpublished datasets, messages were posted on electronic mailing lists (e.g., SPORTPSY) reaching out to social science researchers. Authors with a background in doping research were also invited via personal email requests to contact us if they had any unpublished information that was relevant to our study.

\subsection{Eligibility Criteria and Study Selection}

Studies that measured doping behaviors and/or doping intentions and at least one other demographic (e.g., age, sex), psychological (e.g., attitudes, morality), or socialcontextual (e.g., participation in team sport, motivational climate) variable were included. Using the above criteria, 104 independent studies were identified. When statistical 
information required for the meta-analysis was not available in the articles retrieved, we contacted the authors in order to request it. Forty one studies were excluded because the corresponding authors either did not reply or were unable to provide the required information. A flow diagram showing the flow of dataset selection is shown in Figure 1. Consequently, the final pool included 63 independent studies [4, 17-19, 21-23, 27-82]. The majority of included studies were journal articles (number of studies, $k=46$ ). Other sources included online datasets $(k=11)$, theses/dissertations $(k=3)$, unpublished manuscripts/datasets $(k=2)$, and a report retrieved from the WADA website $(k=1)$. Most of the included studies were crosssectional $(k=55)$. Far fewer studies used longitudinal/prospective $(k=4)$ or experimental designs $(k=4)$.

\subsection{Data Collection Process/Summary Measures}

Coding of study information was conducted by one author with a sample of studies coded independently and cross-checked by another author. We coded for the type of publication (e.g., published journal article, student thesis, manuscript under review), study design (e.g., cross-sectional, longitudinal/prospective, experimental), background of participants (e.g., competitive athletes, gym users, students), type of sport participants engaged in (e.g., team, individual), and age group of participants (e.g., adolescent, adults). These variables were subsequently tested as potential moderators of effect sizes. The summary measures/effect sizes recorded were odds ratios (for dichotomous variables predicting doping behaviors), zero-order Pearson's correlations (when one or both variables were interval or continuous), and Cohen's $d$ (for intervention versus control group comparisons). Cronbach's alphas were also recorded to correct for measurement error in the meta-analyzed effect sizes [83]. A summary of the information coded in each included study is presented in Electronic Supplementary Material Appendix S2. 


\subsection{Assessment of Quality/Risk of Bias}

To ensure study quality would not lead to biases in results, the quality of included studies was assessed [84]. The assessment tool developed by Higgins et al. [84] was adopted in this study. However, as this tool was designed for studies using an experimental design only, we created other criteria (see Electronic Supplementary Material Appendix S3) for cross-sectional (e.g., whether valid measures were used) and longitudinal/prospective studies (e.g., whether dropout from the study was random). For each criterion, included studies were rated as having 'low risk' or 'high risk' of bias. A study was deemed to have low risk of bias if it was rated as having 'low risk' for all assessed criteria. The remaining studies (i.e., those with one or more criteria being considered as indicating 'high risk') were deemed as having potential risk of biases. We tried to eliminate risk of bias across studies by actively seeking for unpublished datasets and by calculating the fail-safe numbers $\mathrm{N}$ statistic (see section 2.6).

To determine whether the assessment criteria were appropriate, two of the authors independently rated the study quality of three studies (one cross-sectional, one longitudinal/prospective, and one experimental). The assessment ratings were compared; out of 29 ratings given for the three studies, only one was different, and this disagreement was resolved after a discussion. The rationales for giving the ratings for each subcategory were also discussed between the two raters to ensure that ratings were given based on similar evidence or reasons. The assessments of all other studies included in the meta-analyses were then conducted by one of the two authors.

\subsection{Synthesis of Results}

The random effect methods proposed by Shadish and Haddock [85] were used to meta-analyze odds ratios (OR). The procedures suggested by Hunter and Schmidt [83], which 
also adopt a random effect model, were used for Pearson's correlations and Cohen's $d$. The population effect size $(\mathrm{OR}$, correlation $[\rho]$, or standardized difference $[\delta]$, respectively) was calculated for each meta-analyzed relation, adjusting for sampling error (by taking into account the sample size). When computing effect sizes, attenuation due to measurement error was corrected using Cronbach's alphas. As Cronbach's alphas were not available for all recorded effect sizes, the artifact distribution meta-analytical procedures [83] were employed. Further, 95\% confidence intervals $(95 \% \mathrm{CI})$ were generated for all meta-analyzed effect sizes. If the $95 \% \mathrm{CI}$ of an effect size did not encompass the point estimate representing a null effect (i.e., 1 for OR, 0 for $\rho$ and $\delta$ ), the effect was considered to be real. When a real effect was found, the magnitude of the meta-analyzed effect sizes was labeled using the criteria suggested by previous researchers $[86,87]$. Essentially, an OR with a value between 1.68 to 3.47 was considered small, 3.47 to 6.71 medium, and above 6.71 large (the reciprocals of these cut-off values were used when $\mathrm{OR}<1$ ). The cut-off values used to label small, medium, and large correlations were $0.1,0.3$, and, 0.5 , respectively; for standardized mean differences, we used values of $0.2,0.5$, and 0.8 , respectively.

\subsection{Additional Analyses}

To address the issue of possible publication bias in the literature [88], we calculated the 'fail-safe numbers' (FSNs) when small to medium, medium, or large effects were found. A FSN represents the number of studies with null findings which, if included in the metaanalysis, would reduce the effect to a small size (i.e., 1.68 for OR, 0.1 for correlation, and 0.2 for standardized differences). The formula provided by Hunter and Schmidt [83] was used to derive the FSNs for the meta-analyzed effect sizes. If the FSN corresponding to an effect size is large, it is considered unlikely that the effect found is due to publication bias.

To test for heterogeneity in the obtained effect sizes, the $I^{2}$ statistic [89] was used. Specifically, the $I^{2}$ is an estimate of the percentage of differences in combined effects sizes 
which is due to true heterogeneity (i.e., not due to chance). A smaller $I^{2}$ suggests that effect sizes are more consistent across studies. Effect sizes with $I^{2}$ values smaller than $25 \%$ were considered to be homogeneous [89]. When $I^{2}$ values exceeded $25 \%$, heterogeneity was suggested and moderator analyses were conducted. In essence, separate sub-group effect sizes were calculated for each level of the potential moderator (e.g., separate effect sizes for the moral disengagement-doping intention relationship were calculated for different types of study design). A variable was considered to be a moderator if the $95 \%$ CIs of any two subgroup effect sizes did not overlap [90]. The potential moderators we tested in this study were: sex of participants (males versus females), publication status (published $[k=46]$ versus unpublished $[k=17]$ ), study design (cross-sectional $[k=55]$ versus longitudinal/prospective $[k=4]$ versus experimental $[k=4]$ ), background of participants (competitive athletes $[k=16]$ versus gym users $[k=12]$ versus students $[k=35]$ ), type of sport (team sports $[k=4]$ versus individual sports $[k=11]$ versus a mixture of both $[k=21]$ ), age group of participants (adults $[k=22]$ versus adolescents $[k=29]$ versus a mixture of both $[k=10])$, and study quality (studies with low risk of bias $[k=55]$ versus studies with potential risk of bias $[k=8]$ ).

Using the meta-analyzed effect sizes we conducted a path analysis of a model based on the TPB [13]. Path analysis and meta-analysis can complement each other [91, 92]. Path analysis can capture interdependencies between variables that meta-analysis cannot capture, because the latter can look at the relation of only two variables at a time. On the other hand, meta-analysis removes the effects of artifacts (i.e., sampling error) from data before path analysis. Hence, our path analysis takes the two-variable associations of the TPB variables established by the meta-analysis and tests them in a multiple variable context.

\section{Results}

The results of all meta-analyzed effect sizes are presented in Table 1. A glossary of the specialized terms in this table is provided in Electronic Supplementary Material Appendix 
S4. Effect sizes from a single study are shown in the tables but will not be interpreted further. Where moderator effects were found, the sub-group effect sizes are presented in Table 2.

\subsection{Effect Sizes of Experimental Studies Comparing Intervention and Control Groups}

With respect to doping behaviors, we compared the differences in numbers of new reported cases of doping between the intervention and control groups over a sport season/ school year. We found that the interventions did not show a real effect in terms of reducing doping behaviors $(\mathrm{OR}=0.76,95 \% \mathrm{CI}[0.27,2.17]$; $\mathrm{OR}<1$ indicates there were fewer reports of new doping behaviors in intervention groups, but the CI included 1). In terms of doping intentions, standardized difference scores between intervention and control groups were meta-analyzed. Over the same period of time, the interventions showed a very small reduction in doping intentions $(\delta=-0.12,95 \%$ CI $[-0.13,-0.11])$. Nonetheless, few studies $(k$ $=3$ and $k=2$ for behaviors and intention, respectively) were included in these analyses (hence why forest plots for these studies are not presented), and therefore the results should be interpreted with caution.

\subsection{Effect Sizes of Demographic and Social-Contextual Variables Predicting Doping Behaviors/Doping Intentions}

Demographic and social-contextual variables were examined in conjunction with doping behaviors and/or intentions in the included pool of non-experimental studies. In terms of demographic variables, we found that males reported more doping behaviors than females $(\mathrm{OR}=2.72,95 \% \mathrm{CI}[2.16,3.42])$. We also found that doping behaviors were more prevalent in people who had friends that doped, compared to those who did not (OR $=6.40,95 \% \mathrm{CI}$ $[3.46,11.84])$. The use of legal supplements was related to more doping behaviors $(\mathrm{OR}=$ $8.24,95 \%$ CI $[5.07,13.39])$ and higher levels of doping intentions ( $\rho=0.36,95 \%$ CI $[0.20$, $0.52])$. Age was also found to be related to doping intentions ( $\rho=0.05,95 \%$ CI $[0.02,0.09])$. 
However, the size of this effect is very small.

In terms of moderator analyses, we found that the background of participants moderated the effect between sex and doping behaviors. Specifically, we found that this effect was stronger for gym users $(\mathrm{OR}=7.77,95 \% \mathrm{CI}[5.31,11.37])$ than competitive athletes $(\mathrm{OR}=2.17,95 \% \mathrm{CI}[1.16,4.08])$ and students $(\mathrm{OR}=2.48,95 \% \mathrm{CI}[2.04,3.00])$. For the relation between age and doping behaviors, a very small negative effect was found in female $(\rho=-0.03,95 \%$ CI $[-0.05,-0.02])$, but not in male participants $(\rho=0.005,95 \%$ CI $[-0.01,0.02])$. Another moderator of the association between age and doping behaviors was the background of participants. Specifically, we found a very small effect between these variables in gym users $(\rho=0.09,95 \%$ CI $[0.01,0.17])$, but not in students $(\rho=-0.01,95 \%$ CI $[-0.02,0.003])$ as the $95 \%$ CIs did not overlap. Further, studies with low risks of bias showed a very small negative effect of age on doping behaviors ( $\rho=-0.01,95 \%$ CI $[-0.02,-0.001])$, but those with potential risk of bias reported a small positive effect $(\rho=0.14,95 \%$ CI $[0.07$, 0.21]). We also found that study design was a moderator of the age - doping intentions relation. Although a very small effect was found in both cross-sectional studies $(\rho=0.07$, 95\% CI $[0.04,0.11])$ and longitudinal/prospective studies $(\rho=0.003,95 \%$ CI $[0.002,0.004])$, their corresponding 95\% CIs did not overlap, indicating that these effect sizes were not equivalent.

\subsection{Effect Sizes of Psychological Variables Predicting Doping Behaviors/Doping}

\section{Intentions}

Some of the effect sizes of psychological variables predicting doping behaviors and intentions were based on a very small number of studies. Consequently, moderator analyses could not be conducted because when these effect sizes were broken down to moderator subgroups, there was insufficient number of studies for all subgroups to allow meaningful 
comparisons. To allow more meaningful comparisons across different levels of moderators, two more stages of analyses were conducted, after the initial stage that calculated an effect size for each psychological variable. In stage two, we collapsed the effect sizes of variables that are conceptually similar (e.g., self-efficacy to refrain from doping and perceived behavioral control; see Table 1 for details regarding how variables were combined) with appropriate reversing (e.g., moral disengagement was reversed when combining effect sizes of morality variables). If conceptually similar constructs were measured in the same study, the weighted averages of the coefficients of interest were used to form a single effect size from this study for the meta-analysis. For brevity reasons, we report below the findings from Stages 2 and 3 only, but the results from all stages are shown in Table 1.

With respect to doping behaviors, we found positive effects from intentions ( $\rho=0.38$, $95 \%$ CI $[0.21,0.55])$, attitudes $(\rho=0.17,95 \%$ CI $[0.04,0.29])$, norms $(\rho=0.36,95 \%$ CI $[0.27,0.45])$, and amotivation $(\rho=0.17,95 \% \mathrm{CI}[0.07,0.26])$. The sizes of these effects were small to medium. Negative effects on doping behaviors were also found from self-efficacy to refrain from doping $(\rho=-0.12,95 \%$ CI $[-0.21,-0.02])$, morality $(\rho=-0.21,95 \%$ CI $[-0.32$, $-0.10])$, autonomous motivation $(\rho=-0.06,95 \%$ CI $[-0.09,-0.03])$, and task achievement goal orientation $(\rho=-0.09,95 \%$ CI $[-0.17,-0.01])$. The effects of the latter two variables though were small. The FSN for the effect between self-efficacy to refrain from doping and doping behaviors was $1(k=5)$, suggesting that only one study with null findings was need to reduce this effect to what is considered a small effect (i.e., reduce it from $\rho=-0.12$ to $\rho=-0.10$ ).

In terms of predicting doping intentions, large positive effects were found for attitudes $(\rho=0.52,95 \%$ CI $[0.44,0.60])$ and norms $(\rho=0.53,95 \%$ CI $[0.43,0.63])$. Small effects were found for dissatisfaction with appearance/body image ( $\rho=0.20,95 \%$ CI $[0.12,0.29])$, amotivation $(\rho=0.24,95 \%$ CI $[0.20,0.27])$, and ego achievement goal orientation $(\rho=0.14$, $95 \%$ CI $[0.09,0.20])$. However, the effect size between doping intentions and ego 
achievement goal orientation has a FSN of $2(k=4)$. Small to moderate negative effects were also found from self-efficacy to refrain from doping ( $\rho=-0.27,95 \%$ CI $[-0.41,-0.14])$ and morality $(\rho=-0.31,95 \%$ CI $[-0.47,-0.16])$. An effect was also found from task achievement goal orientation $(\rho=-0.08,95 \%$ CI $[-0.14,-0.02])$. Nonetheless, the size of this latter effect is small.

For the third stage of the analyses, variables were categorized as either a positive or negative correlates of doping intention or behaviors. A variable was categorized as a positive correlate if a real positive effect with doping intention/behaviors was found in the previous stages. In contrast, variables with real negative effects were considered as negative correlates. Based on this categorization, we collapsed the effect sizes associated with all positive and negative correlates and conducted meta-analyses and moderator analyses using the combined effect sizes. The variables included in these analyses are shown in Table 1. Forest plots representing the third stage of meta-analyses are presented in Electronic Supplementary Material Appendix S5.

We found that the effect sizes associated with the positive $(\rho=0.20,95 \%$ CI $[0.08$, $0.32])$ and negative $(\rho=-0.13,95 \%$ CI $[-0.19,-0.07])$ correlates were small to medium. The FSN for the effect between doping behaviors and negative correlates was also small $(\mathrm{FSN}=4$, $k=11$ ), suggesting that a relatively small number of studies (i.e., 4) with null findings would bring the meta-analyzed effect to a small value. With respect to doping intentions, we found a medium effect from positive $(\rho=0.44,95 \%$ CI $[0.36,0.51])$ and negative $(\rho=-0.28,95 \%$ CI $[-0.39,-0.17])$ correlates.

In this third stage of the analysis we also found some moderation effects. For the positive correlates - doping behaviors relation, a medium effect size was found when only studies with low risk of bias were included $(\rho=0.31,95 \%$ CI $[0.21,0.41])$. However, a small negative effect was found in studies that were rated to have potential risks of bias $(\rho=-0.10$, 
$95 \% \mathrm{CI}[-0.13,-0.06])$. We also found that the relation between positive correlates and doping intentions was moderated by publication status and study design. Specifically, we found that the effects in published datasets $(\rho=0.49,95 \%$ CI $[0.40,0.59])$ were stronger than those found in unpublished datasets $(\rho=0.31,95 \%$ CI $[0.24,0.39])$. Nonetheless, the size of both effects was medium. In terms of study design, we found a medium effect for crosssectional studies $(\rho=0.40,95 \%$ CI $[0.31,0.48])$, but a large effect for longitudinal/perspective studies $(\rho=0.56,95 \%$ CI $[0.52,0.61])$. Study design also moderated the negative correlates-doping intentions relation. A small effect was found in cross-sectional studies $(\rho=-0.21,95 \%$ CI $[-0.33,-0.10])$, but the magnitude of the effect was stronger in longitudinal/prospective studies ( $\rho=-0.44,95 \%$ CI $[-0.45,-0.43])$. Finally, we found that the effect size between negative correlates and doping intentions was small in competitive athletes $(\rho=-0.16,95 \%$ CI $[-0.29,-0.02])$, but large in gym users $(\rho=-0.62,95 \%$ CI $[-0.85$, $-0.40])$.

\subsection{Path Analyses of a Theory of Planned Behavior Model}

The TPB $[13,20]$ was the most frequently utilized theoretical framework within the pool of included studies. Thus, in order to examine the inter-relation between all TPB constructs and doping behaviors and intentions, we conducted path analyses using metaanalyzed effect sizes from the second stage of our analyses. Path analyses were conducted using the Mplus 6.1 software [93]. Following recommendations by Viswesvaran and Ones [92], the harmonic mean of the sample sizes underpinning the corresponding effect sizes of the correlation matrix was used as the total sample size $(n=5,046)$ for the tested models. Based on the model proposed by Ajzen [13], we tested an initial model with attitudes, norms, and self-efficacy to refrain from doping predicting intentions to dope; the latter, in turn, predicted doping behaviors. We used self-efficacy to refrain from doping instead of perceived 
behavioral control as they both reflect efficacy beliefs. Further, past evidence has shown that self-efficacy to refrain from doping is a stronger predictor of doping intentions [16] and, in contrast to perceived behavioral control, a significant predictor of doping intentions in both users and non-users [94]. This model did not fit well [95]: $\chi^{2}(3)=288.98, p<0.001$, comparative fit index $(\mathrm{CFI})=0.92$, root mean square error of estimation $(\mathrm{RMSEA})=0.14$, standardized root mean square residual $(\mathrm{SRMR})=0.04$.

We tested three other plausible alternative models by freeing direct paths from attitudes, norms, and self-efficacy to refrain from doping (only one of these paths was freed in each alternative model) to doping behaviors. A plausible model was accepted if the improvement in CFI was larger than 0.01 compared to the initial model [96]. The alternative models with direct paths from attitudes or self-efficacy to refrain from doping to behaviors did not meet this criterion and were rejected. However, the model with a direct path from norms to doping behaviors (see Figure 2) was accepted (change in CFI $=0.060$ ). This final model had a significant $\chi^{2}$, but other fit indices suggested an acceptable model fit: $\chi^{2}(2)=$ 78.35, $p<0.001, \mathrm{CFI}=0.98, \mathrm{RMSEA}=0.09, \mathrm{SRMR}=0.02$. In this model, the direction of paths from attitudes $(\beta=0.31)$, norms $(\beta=0.34)$, and self-efficacy to refrain from doping ( $\beta$ $=-0.14$ ) to doping intentions were in the expected direction (all path $p<0.001$ ). Paths from norms $(\beta=0.22, p<0.001)$ and intention $(\beta=0.26, p<0.001)$ to doping behaviors were also positive. Indirect effects on doping behaviors from attitudes $(\beta=0.12)$ and self-efficacy to refrain from doping $(\beta=-0.06)$ were also significant at $p<0.001$.

\section{Discussion}

This article represents the first meta-analysis within the doping literature. Its primary aim was to synthesize data from independent samples in an effort to identify key demographic, psychological, and socio-contextual factors that are related to doping intentions 
and doping behaviors. In sum, our findings corroborate Petróczi and Aidman's [97] argument that decisions to dope reflect a complex interplay of multiple factors and may not be accurately explained by a few variables alone. This is a notable conclusion when one considers that although a variety of social cognitive models purporting to delineate the key determinants of behavior exist, the TPB has been the most widely tested theory in the context of doping in sport. Moreover, for some of the identified predictor variables there was only a limited pool of studies available. Consequently, the findings with regard to these variables must be interpreted with caution, including findings from experimental studies. Nevertheless, collapsing the variables in the final stage analysis of positive and negative correlates of doping intention and behaviors gives some indication of the magnitude of the statistical effects, which were small to medium. This meta-analysis reconfirms the conclusion drawn by Backhouse and colleagues [25] that the evidence base on the psycho-social predictors of doping intentions and behaviors is limited by span and methodology. This absence of evidence continues to be an important consideration for policy makers and developers of antidoping prevention programs.

\subsection{Effect of interventions on doping intentions and behavior}

We firstly evaluated the effectiveness of existing randomized controlled trials (RCTs) and only four experimental studies met the eligibility criteria for inclusion in our metaanalysis. The four studies presented data from the evaluations of two long-standing North American prevention programs, namely the ATLAS [42, 98, 99], and ATHENA [26, 99] programs. The ATLAS/ATHENA-based RCTs included in our analyses showed a very small but significant reduction in doping intentions but no changes in doping behavior. This might be attributed to the content of the ATLAS and ATHENA interventions. Both tackle doping alongside other behaviors, such as healthy eating and training regimes. Perhaps the lack of a 
clear focus on doping explains the small effect on intentions and the non-significant effect on actual behavior. In particular, ATHENA is largely focused on information and activities about healthy and disordered eating, drug use (i.e., alcohol, tobacco, marijuana, and anabolic steroids), monitoring nutritional intake, and improving psychological factors, such as depression and mood $[26,67,100]$. Hence, both interventions aim to influence adolescent athletes'/students' overall health-related behaviors, and this may happen at the expense of a greater focus on doping use. Furthermore, it is possible that the small effects found in the ATLAS/ATHENA interventions reflect floor effects: participants' doping intentions at baseline (pre-intervention) were low. Therefore, there was not much room for a further reduction in intentions and behavior. For example, in Goldberg et al.'s [98] study, preintervention intentions to use steroids were 5.7 and 6.3 in the experimental and control groups respectively (1-7 scale with 7 being no intent to use).

\subsection{Effect sizes of demographic and social-contextual variables}

Effect sizes from non-experimental studies were also included in the present metaanalysis. We looked at the effect sizes associated with demographic and socio-contextual variables. Males and gym users were more likely to report doping use compared to females and competitive athletes or students. Further, the use of legal nutritional supplements and being affiliated with peers who use doping substances, predicted greater self-reported doping use. Use of legal supplements had a large positive effect on doping behavior. This suggests that users of legal supplements are at a much higher risk of using illegal drugs. This finding is notable when one considers the widespread use of legal supplements across all levels of sport [101-104]. In a recent study, Backhouse et al. [23] suggested that a gradual increase in nutritional supplements' consumption served as a gateway to doping use. Specifically, in a sample of competitive athletes, Backhouse and colleagues found that doping use was threeand-a-half times more prevalent in nutritional supplement users as compared to nonusers. 
This finding was accompanied by significant differences in doping attitudes and beliefs between nutritional supplement users and nonusers. A potential explanation is that the habitual use of otherwise legal nutritional supplements familiarizes athletes with the notion of chemically-assisted performance enhancement [97] and this can further facilitate transitions to doping use. In a similar vein, Tsorbatzoudis et al. [105] indicated that nutritional supplement users are two to three times more likely to report doping use and they also displayed biased normative beliefs related to doping use (i.e., they perceived doping as more prevalent in fellow athletes and socially approved).

\subsection{Effects of psychological variables}

The present meta-analysis also investigated the effects of psychological variables on doping intentions and behavior. Specifically, task goal orientation was inversely related to doping use, whereas ego goal orientation and controlled motivation were positively related. These findings suggest that ego-oriented and externally regulated athletes display a more maladaptive set of behavioral responses. These athletes are more inclined to dope and this should be taken into account when developing prevention education interventions.

Many of the included studies utilized TPB but expanded on this framework by utilizing their own measures and incorporating additional distal predictors of doping behavior. The meta-analysis showed that pro-doping attitudes and biased normative beliefs (i.e., overestimating the actual prevalence and social acceptability of doping use) directly and positively predicted doping intentions. Accordingly, higher scores in self-efficacy to resist temptations and anticipated regret inversely predicted doping intentions and use. These findings imply that the TPB - and its extensions - offer a useful framework for understanding the decision making process towards doping use.

Having said this, it is important to acknowledge that the TPB is a highly rational 
model and Ajzen [106] argues that it does not adequately account for the cognitive and affective processes which bias judgments and behavior. Stewart and Smith [107] have argued that athletes' decisions may not always rest on rational thinking. This may also apply to intentions to dope which might be based on unconscious factors which bias judgment. Further, doping use might be un-intentional via the consumption of contaminated nutritional supplements [108]. Therefore, the application of alternative models and methods is encouraged in this field in order to further our understanding of the complex doping phenomenon. Further, new approaches have been proposed suggesting that doping use is influenced by the interplay of sociocultural, socioeconomic, and personality factors. For instance, Donovan et al. [109], Petroczi and Aidman [97], and Strelan and Boeckmann [110] discussed the role of personality factors, decisions regarding the pros and cons of doping use, the legislative system, the affordability, and availability of prohibited substances. Stewart and Smith [107] provided a macro-analysis of doping use in sport suggesting that, alongside to personality factors, globalization and commercialization of sport, and sport cultures are key factors in explaining doping use. However, research testing these ideas is rather scarce and future studies which take into account these variables are encouraged [107].

In the current study higher levels of sportspersonship and moral disengagement negatively predicted doping intentions and doping use. These findings highlight the important role of morality in preventing doping use. Finally, dimensions of body image, more specifically, concerns about muscularity and thinness, and overall dissatisfaction with body appearance were found to significantly relate to doping intentions and doping use. These findings suggest that maladaptive perceptions of body image may facilitate doping use. This could be through the use of illegal substances as these can be used as a strategy to augment or reduce an individual's physique. Therefore, body image should be targeted in prevention programs, particularly those that reach adolescent athletes. 
In this meta-analysis we also examined whether several factors moderated the reported effect sizes. Although some of the effects varied in size across different levels of moderators, in general, the direction of the effects remained unchanged. However, we found that the effect sizes from some studies deemed as having potential biases may alter the directions of the effect in an unexpected way (e.g., a negative effect between positive correlates of doping and doping behaviors). Further, published studies reported bigger effect sizes than unpublished ones. These findings, therefore, emphasize the importance of eliminating possible sources of biases when conducting empirical research in this field to avoid dubious results.

\subsection{Path Analysis}

Using the meta-analyzed effect sizes, we also tested a path model based on the TPB. We found that the paths from attitudes, perceived norms, and self-efficacy to refrain from doping, were significant predictors of doping intentions, and in the expected direction. Furthermore, a positive path from doping intentions to doping behaviors was found. By comparing alternative nested models, we also found that perceived norms had a direct effect on doping behaviors. These results are slightly unexpected because in the TPB literature perceived norms are often found to be the weakest, or even non-significant, predictor of intentions $[111,112]$. However, unlike many non-doping related TPB studies that assessed social norms through subjective norms (i.e., perceived social approval of doping), many of the studies we analyzed assessed other aspects of social norms, such as descriptive norms (i.e., what significant others themselves do). This encompassing assessment appears important when one considers the assertion that the role of norms varies dependent on the type of behavior [113]. 
Overall, the findings of this meta-analysis underscore the importance of normative influences on doping use. Normative influences to engage in doping can range from mere peer pressure and perceived social acceptability of doping by teammates, to internal and external pressures associated with results and enhanced performance. Hence, the athlete's entourage (e.g., coach and teammates) and perceptions about the prevalence of doping use among other athletes seem to be important determinants of the decision to engage in doping. It is noteworthy that perceptions of social norms do not have to be accurate to be influential [114] and future research should address the cognitive processes (e.g., heuristics) that lead to biased normative beliefs. In order to increase the predictive power of the normative component of the TPB, future research could pay closer attention to other normative variables (e.g., personal, injunctive and moral norms) [112]. For example, it is unclear whether observing the doping behavior of others is of greater motivational importance compared to perceived social pressure from others. Finally, Rivis and Sheeran's [111] observation that young people are particularly susceptible to descriptive norms suggests that interventions with adolescent groups should be bespoke to the needs of that group in order to foster a strong sense of individuality and uniqueness.

\subsection{Limitations}

The most important limitation of this work is that many meta-analyzed effect sizes were based on a small number of studies. This is because the studies conducted in this area have been very broad, perhaps too broad, in terms of identifying demographic, personal, and situational predictors of doping intentions and behavior. Nevertheless, this review represents an initial effort to quantify the psychosocial variables that are most strongly related to doping behaviors or intentions to engage in such behaviors. Also, the current conclusions assume that self-reported behaviors are accurate reflections of people's actions (all included studies used self-reports to measure doping behavior/use). Further, most of the meta-analyzed effect 
sizes between predictors of doping with doping intentions and behaviors were derived from correlational studies; more prospective and intervention studies are needed in this area.

As a consequence, the identified effect sizes show statistical and not causal effects. For example, a large effect size between perceived norms and intentions may also suggest people having stronger intentions to dope would perceive more people to be doping [63]. Similarly, people may report having more positive attitudes towards doping because they intend to use illegal substances. Another limitation was that we were unable to test some potentially important moderator effects. For example, in studies that reported the numbers or percentages of participants using legal substances, these were reported for the total sample and not by subgroups. Therefore, comparing effect sizes for different levels of moderators was not possible, unless authors were willing to provide us with access to their raw data.

\subsection{Practical Implications}

Anti-doping education should target normative influences and attempt to denormalize doping use in sports and exercise settings. Prevention programs are particularly encouraged in the early adolescent years when attitudes are forming [115] and when adolescents tend to be more vulnerable to normative influences. Thus, related interventions should be more intensive for this age group and greater attention should be afforded to peer influence and related socio-emotional processes that facilitate risky behavior under social pressure $[116,117]$. For example, forging a unique and individual identity could be important in this context as adolescents appear particularly susceptible to descriptive norms [111]. Finally, there are experimental studies that have demonstrated powerful effects of normsbased interventions in changing adults' behavior [118, 119]. Doping researchers could utilize these findings and develop norms-based interventions to reduce the risk for doping use.

Future research and preventive guidelines regarding the social environment should also focus on the role of coaches and athlete support personnel in promoting adaptive types of 
motivation (autonomous motivation, task achievement goals). Importantly, future prevention interventions should improve athletes' self-efficacy in resisting temptations (i.e., outcomes of success such as money, fame etc.) which might lead to doping use. In addition, aspects of ethical decision making should be incorporated in interventions designed to tackle doping use. Finally, doping-related interventions should inform athletes on the risks and appropriate use of nutritional supplements. In recent years, contaminated nutritional supplements have become a real cause for concern [108] and at a global level there has been a growing number of claims of inadvertent doping through the use of nutritional supplements. So that athletes can make an informed choice, there is an urgent need to regulate the industry and undertake efficacy trials to assess the performance benefits and health risks associated with the use of these supplements. Furthermore, the use of nutritional supplements may act as a gateway to illegal drug use [23] and this should be acknowledged through increased educational efforts in this area.

\section{Conclusions}

In sum, we found that doping behaviors may be explained by a combination of demographic, social-contextual, and psychological variables. However, the available evidence is still not sufficient in volume and rigorous enough in terms of methodology or diverse enough in terms of its conceptual underpinnings, and this limits our understanding of doping intentions and doping use in sport. Nevertheless, our results support a curriculum shift by placing greater emphasis on modifying norm-based doping perceptions and creating coach and peer task-involving motivational climates. Effective prevention interventions to tackle doping use should target athletes' motivation, cognitions and moral stance, empowering athletes and their entourage to resist unethical temptations and pursue performance improvements through the use of legal substances and methods. 


\section{Acknowledgements}

We would like to thank the World Anti-Doping Agency for funding this project. The funders did not influence this review in any way. The authors have no potential conflicts of interest that are directly relevant to the content of this review. We would also like to thank the following authors and their team (in alphabetical order) for providing the necessary statistical information needed for our meta-analysis: F. Al-Maskari, D. Chan, T. Dodge, E. Donahue, M. Dunn, H. Gabriel, D. Gucciardi, G. Kanayama, F. Lucidi, R. Lugo, K. Miller, A. Moran, S. Nilsson, F. Papadopoulos, A. Petroczi, H. Pope, K. Wiefferink, A. Zelli. 


\section{References}

1. WADA. World anti-doping code. Montreal, Canada: World Anti-Doping Agency; 2009.

2. Baron DA, Martin DM, Magd SA. Doping in sports and its spread to at-risk populations: An international review. World Psychiatry. 2007;6(2):118-23.

3. Pitsch W, Emrich E. The frequency of doping in elite sport: Results of a replication study. Int Rev Sociol Sport. 2011;47(5):559-80.

4. Dunn M, Mazanov J, Sitharthan G. Predicting future anabolic-androgenic steroid use intentions with current substance use: Findings from an internet-based survey. Clin J Sport Med. 2009;19(3):222-7.

5. Blouin AG, Goldfield GS. Body image and steroid use in male bodybuilders. Int J Eat Disord. 1995;18(2):159-65.

6. Laure P. Epidemiologic approach of doping in sport. A review. J Sports Med Phys Fitness. $1997 ; 37(3): 218-24$.

7. Lentillon-Kaestner V, Ohl F. Can we measure accurately the prevalence of doping? Scand J Med Sci Sports. 2011;21(6):e132-e42.

8. Uvacsek M, Nepusz T, Naughton DP, et al. Self-admitted behavior and perceived use of performance-enhancing vs psychoactive drugs among competitive athletes. Scand J Med Sci Sports. $2011 ; 21(2): 224-34$.

9. Petróczi A, Aidman EV, Nepusz T. Capturing doping attitudes by self-report declarations and implicit assessment: A methodology study. Subst Abuse Treat Prev Policy. 2008;3:9. 10. Sottas P-E, Robinson N, Fischetto G, et al. Prevalence of blood doping in samples collected from elite track and field athletes. Clin Chem. 2011;57(5):762-9.

11. Fahey J. WADA in 2013. In: WADA Media Symposium. 2013. http://www.wadaama.org/Documents/News Center/Speeches Presentations/WADA-2013-MediaSymposium-President-Speech.pdf. Accessed 4 Jun 2014. 
12. Working Group on the (in)effectiveness of testing. Report to WADA Executive Committee on lack of effectiveness of testing programs. 2013.

13. Ajzen I. The theory of planned behavior. Organ Behav Hum Decis Process. 1991;50(2):179-211.

14. Fishbein M, Cappella JN. The role of theory in developing effective health communications. J Commun. 2006;56(s1):S1-S17.

15. Goulet C, Valois P, Buist A, et al. Predictors of the use of performance-enhancing substances by young athletes. Clin J Sport Med. 2010;20(4):243-8.

16. Lazuras L, Barkoukis V, Rodafinos A, et al. Predictors of doping intentions in elite-level athletes: a social cognition approach. J Sport Exerc Psychol. 2010;32(5):694-710.

17. Lucidi F, Zelli A, Mallia L, et al. The social-cognitive mechanisms regulating adolescents' use of doping substances. J Sports Sci. 2008;26(5):447-56.

18. Wiefferink CH, Detmar SB, Coumans B, et al. Social psychological determinants of the use of performance-enhancing drugs by gym users. Health Educ Res. 2008;23(1):70-80. 19. Zelli A, Mallia L, Lucidi F. The contribution of interpersonal appraisals to a socialcognitive analysis of adolescents' doping use. Psychol Sport Exerc. 2010;11(4):304-11. 20. Fishbein M. An integrative model for behavioral prediction and its application to health promotion. In: DiClemente RJ, Crosby RA, Kegler MC, editors. Emerging theories in health promotion practice and research. 2nd ed. New York: John Wiley \& Sons; 2009. pp. 215-34. 21. Barkoukis V, Lazuras L, Tsorbatzoudis H, et al. Motivational and sportspersonship profiles of elite athletes in relation to doping behavior. Psychol Sport Exerc. 2011;12(3):20512.

22. Dodge TL, Jaccard JJ. The effect of high school sports participation on the use of performance-enhancing substances in young adulthood. J Adolesc Health. 2006;39(3):367-73. 23. Backhouse SH, Whitaker L, Petroczi A. Gateway to doping? Supplement use in the 
context of preferred competitive situations, doping attitude, beliefs, and norms. Scand J Med Sci Sports. 2013;23(2):244-52.

24. Whitaker L, Long J, Petróczi A, et al. Using the prototype willingness model to predict doping in sport. Scand J Med Sci Sports. in press.

25. Backhouse S, McKenna J, Robinson S, et al. International literature review: Attitudes, behaviors, knowledge and education - drugs in sport: Past, present and future. Report to the World Anti-Doping Agency (WADA). 2007.

26. Elliot DL, Goldberg L, Moe EL, et al. Long-term outcomes of the ATHENA (Athletes Targeting Healthy Exercise \& Nutrition Alternatives) program for female high school athletes. J Alcohol Drug Educ. 2008;52(2):73-92.

27. Chng CL, Moore A. A study of steroid use among athletes: Knowledge, attitude and use. Health Educ. 1990;21(6):12-7.

28. Centers for Disease Control and Prevention. National Youth Risk Behavior Survey (National High School). 1991.

29. Centers for Disease Control and Prevention. National Youth Risk Behavior Survey (National High School). 1993.

30. Brower KJ, Blow FC, Hill EM. Risk factors for anabolic-androgenic steroid use in men. J Psychiatr Res. 1994;28(4):369-80.

31. Centers for Disease Control and Prevention. National Youth Risk Behavior Survey (National High School). 1995.

32. Centers for Disease Control and Prevention. National Youth Risk Behavior Survey (National College). 1995.

33. Nilsson S. Androgenic anabolic steroid use among male adolescents in Falkenberg. Eur J Clin Pharmacol. 1995;48(1):9-11.

34. Allemeier MF. CIAU athletes' use and intentions to use performance enhancing drugs: A 
study utilizing the theory of planned behaviour [Master's thesis]: The University of British Columbia; 1996.

35. Goldberg L, Elliot D, Clarke GN, et al. Effects of a multidimensional anabolic steroid prevention intervention: The Adolescents Training and Learning to Avoid Steroids (ATLAS) program. JAMA-J Am Med Assoc. 1996;276(19):1555-62.

36. Melia P, Pipe A, Greenberg L. The use of anabolic-androgenic steroids by Canadian students. Clin J Sport Med. 1996;6(1):9-14.

37. Vogels T, Brugman E, Coumans B, et al. Correlates of the use of performance-enhancing drugs among young patrons of gymnasiums in the Netherlands. Drugs (Abingdon Engl). 1996;3(1):39-48.

38. Centers for Disease Control and Prevention. National Youth Risk Behavior Survey (National Alternative High School). 1998.

39. Faigenbaum AD, Zaichkowsky LD, Gardner DE, et al. Anabolic steroid use by male and female middle school students. Pediatrics. 1998;101:e6.

40. Centers for Disease Control and Prevention. National Youth Risk Behavior Survey (National High School). 1999.

41. Kindlundh AMS, Isacson DGL, Berglund L, et al. Factors associated with adolescent use of doping agents: anabolic-androgenic steroids. Addiction. 1999;94(4):543-53.

42. Goldberg L, Mackinnon DP, Elliot DL, et al. The adolescents training and learning to avoid steroids program: Preventing drug use and promoting health behaviors. Arch Pediatr Adolesc Med. 2000;154:332-8.

43. Centers for Disease Control and Prevention. National Youth Risk Behavior Survey (National High School). 2001.

44. Pedersen W, Wichstrøm L. Adolescents, doping agents, and drug use: a community study. J Drug Issues. 2001;31(2):517-42. 
45. Miller KE, Barnes GM, Sabo D, et al. A comparison of health risk behavior in adolescent users of anabolic-androgenic steroids, by gender and athlete status. Sociol Sport J. 2002;19:385-402.

46. Goldberg L, Elliot DL, Mackinnon DP, et al. Drug testing athletes to prevent substance abuse: Background and pilot study results of the SATURN (student athlete testing using random notification) study. J Adolesc Health. 2003;32(1):16-25.

47. Laure P, Lecerf T, Friser A, et al. Drugs, recreational drug use and attitudes towards doping of high school athletes. Int J Sports Med. 2004;25(2):133-8.

48. Lucidi F, Grano C, Leone L, et al. Determinants of the intention to use doping substances: An empirical contribution in a sample of Italian adolescents. Int J Sport Psychol. 2004;35(2):133-48.

49. Centers for Disease Control and Prevention. National Youth Risk Behavior Survey (National High School). 2005.

50. Donahue EG, Miquelon P, Valois $\mathrm{P}$, et al. A motivational model of performanceenhancing substance use in elite athletes. J Sport Exerc Psychol. 2006;28:511-20.

51. Kanayama G, Barry S, Hudson JI, et al. Body image and attitudes toward male roles in anabolic-androgenic steroid users. Am J Psychiatry. 2006;163:697-703.

52. Papadopoulos FC, Skalkidis I, Parkkari J, et al. Doping use among tertiary education students in six developed countries. Eur J Epidemiol. 2006;21(4):307-13.

53. Striegel H, Simon P, Frisch S, et al. Anabolic ergogenic substance users in fitness-sports: A distinct group supported by the health care system. Drug Alcohol Depend. 2006;81(1):11-9. 54. Centers for Disease Control and Prevention. National Youth Risk Behavior Survey (National High School). 2007.

55. Dodge T, Jaccard JJ. Negative beliefs as a moderator of the intention-behavior relationship: Decisions to use performance-enhancing substances. J Appl Soc Psychol. 
2007;37(1):43-59.

56. Elliot DL, Cheong J, Moe EL, et al. Cross-sectional study of female students reporting anabolic steroid use. Arch Pediatr Adolesc Med. 2007;161:572-7.

57. Laure P, Binsinger C. Doping prevalence among preadolescent athletes: a 4-year followup. Br J Sports Med. 2007;41(10):660-3.

58. Petróczi A. Attitudes and doping: A structural equation analysis of the relationship between athletes' attitudes, sport orientation and doping behaviour. Subst Abuse Treat Prev Policy. 2007;2:34.

59. Wanjek B, Rosendahl J, Strauss B, et al. Doping, drugs and drug abuse among adolescents in the state of Thuringia (Germany): Prevalence, knowledge and attitudes. Int J Sports Med. 2007;28(4):346-53.

60. Al-Falasi O, Al-Dahmani K, Al-Eisaei K, et al. Knowledge, attitude and practice of anabolic steroids use among gym users in Al-Ain district, United Arab Emirates. Open Sp Med J. 2008;2:75-81.

61. Dodge T, Jaccard JJ. Is abstinence an alternative? Predicting adolescent athletes' intentions to use performance enhancing substances. J Health Psychol. 2008;13(5):703-11. 62. Moran A, Guerin S, Kirby K, et al. The development and validation of a doping attitudes and behaviour scale. Report to World Anti-Doping Agency and The Irish Sports Council. 2008 .

63. Petroczi A, Mazanov J, Nepusz T, et al. Comfort in big numbers: Does over-estimation of doping prevalence in others indicate self-involvement? J Occup Med Toxicol. 2008;3:19.

64. Rees CR, Zarco EPT, Lewis DK. The Steroids/Sports Supplements Connection: Pragmatism and Sensation-Seeking in the Attitudes and Behavior of JHS and HS Students on Long Island. J Drug Educ. 2008;38(4):329-49.

65. Centers for Disease Control and Prevention. National Youth Risk Behavior Survey 
(National High School). 2009.

66. Lugo R. Sports and health: The influence of motivational orientation on body image and doping behaviors [Master's thesis]: Universitet I Oslo; 2009.

67. Ranby KW, Aiken LS, Mackinnon DP, et al. A mediation analysis of the ATHENA intervention for female athletes: prevention of athletic-enhancing substance use and unhealthy weight loss behaviors. J Pediatr Psychol. 2009;34(10):1069-83.

68. Rodek J, Sekulic D, Pasalic E. Can we consider religiousness as a protective factor against doping behavior in sport? J Relig Health. 2009;48(4):445-53.

69. Ip EJ, Barnett MJ, Tenerowicz MJ, et al. Women and anabolic steroids: An analysis of a dozen users. Clin J Sport Med. 2010;20(6):475-81.

70. Thorlindsson T, Halldorsson V. Sport, and use of anabolic androgenic steroids among Icelandic high school students: A critical test of three perspectives. Subst Abuse Treat Prev Policy. 2010;5:32.

71. Zelli A, Lucidi F, Mallia L. The relationships among adolescents' drive for muscularity, drive for thinness, doping attitudes, and doping intentions. J Clin Sp Psych. 2010;4:39-52. 72. Centers for Disease Control and Prevention. National Youth Risk Behavior Survey (National High School). 2011.

73. Gucciardi DF, Jalleh G, Donovan RJ. An examination of the sport drug control model with elite Australian athletes. J Sci Med Sport. 2011;14(6):469-76.

74. Neeraj K, Maman P, Sandhu JS. Why players engage in drug abuse substances? A survey study. Doping J. 2011;8:1.

75. Petroczi A, Uvacsek M, Nepusz T, et al. Incongruence in doping related attitudes, beliefs and opinions in the context of discordant behavioural data: in which measure do we trust? PLoS ONE. 2011;6:e18804.

76. Uvacsek M, Nepusz T, Naughton DP, et al. Self-admitted behavior and perceived use of 
performance-enhancing vs psychoactive drugs among competitive athletes. Scand J Med Sci Sports. 2011;21(2):224-34.

77. Allahverdipour H, Jalilian F, Shaghaghi A. Vulnerability and the intention to anabolic steroids use among Iranian gym users: An application of the theory of planned behavior. Subst Use Misuse. 2012;47(3):309-17.

78. Pope Jr. HG, Kanayama G, Hudson JI. Risk factors for illicit anabolic-androgenic steroid use in male weightlifters: A cross-sectional cohort study. Biol Psychiatry. 2012;71(3):254-61. 79. Barkoukis V, Lazuras L, Tsorbatzoudis H. Beliefs about the causes of success in sports and susceptibility for doping use in adolescent athletes. J Sports Sci. 2013.

80. Chan DKC, Hagger MS, Hardcastle SJ, et al. Behavioural, normative, and control beliefs of doping avoidance: A perspective from the theory of planned behaviour [unpublished]. 2013.

81. Tsorbatzoudis H, Barkoukis V, Lazuras L. Relation between doping behaviours and psychological variables [unpublished]. 2013.

82. Whitaker L. Applying the prototype willingness model to doping in sport [Doctoral thesis]: Leeds Metropolitan University; 2013.

83. Hunter JE, Schmidt FL. Methods of meta-analysis: correcting error and bias in research findings. 2nd ed. Thousand Oaks, CA: Sage Publications, Inc.; 2004.

84. Higgins JPT, Altman DG, Sterne JAC. Chapter 8: Assessing risk of bias in included studies. In: Higgins JPT, Green S, editors. Cochrane handbook for systematic reviews of interventions: The Cochrane Collaboration; 2011.

85. Shadish WR, Haddock CK. Combining estimates of effect size. In: Cooper H, Hedges LV, editors. The handbook of research synthesis. New York, NY: Russell Sage Foundation; 1994. pp. 261-81.

86. Chen $\mathrm{H}$, Cohen $\mathrm{P}$, Chen $\mathrm{S}$. How big is a big odds ratio? Interpreting the magnitudes of 
odds ratios in epidemiological studies. Commun Stat Simul Comput. 2010;39(4):860-4.

87. Cohen J. Statistical power analysis for the behavioral sciences. revised ed. New York: Academic Press; 1977.

88. Rosenthal R. The "file drawer problem" and tolerance for null results. Psychol Bull. 1979;86(3):638-41.

89. Higgins JPT, Thompson SG, Deeks JJ, et al. Measuring inconsistency in meta-analyses. BMJ. 2003;327:557.

90. Hwang MI, Schmidt FL. Assessing moderating effect in meta-analysis: A re-analysis of top management support studies and suggestions for researchers. Eur J Inf Syst. 2011;20(6):693-702.

91. Landis RS. Successfully combining meta-analysis and structural equation modeling: Recommendations and strategies. J Bus Psychol. 2013;28(3):251-61.

92. Viswesvaran C, Ones DS. Theory testing: Combining psychometric meta-analysis and structural equations modeling. Pers Psychol. 1995;48(4):865-85.

93. Muthén B, Muthén L. Mplus user guide. Los Angeles, CA: Muthén and Muthén; 2008. 94. Barkoukis V, Lazuras L, Tsorbatzoudis H, et al. Motivational and social cognitive predictors of doping intentions in elite sports: An integrated approach. Scand J Med Sci Sports. 2013;23(5):e330-40.

95. Hu L-t, Bentler PM. Cutoff criteria for fit indexes in covariance structure analysis: Conventional criteria versus new alternatives. Struct Equ Modeling. 1999;6(1):1-55. 96. Cheung GW, Rensvold RB. Evaluating goodness-of-fit indexes for testing measurement invariance. Struct Equ Modeling. 2002;9(2):233-55.

97. Petróczi A, Aidman E. Psychological drivers in doping: The life-cycle model of performance enhancement. Subst Abuse Treat Prev Policy. 2008;3:7. 98. Goldberg L, Elliot DL, Clarke GN, et al. The Adolescents Training and Learning to 
Avoid Steroids (ATLAS) prevention program: Background and results of a model intervention. Arch Pediatr Adolesc Med. 1996;150(7):713-21.

99. Goldberg L, Elliot DL. Preventing substance use among high school athletes: The ATLAS and ATHENA programs. J Appl Sch Psychol. 2005;21(2):63-87.

100. Elliot DL, Goldberg L, Moe EL, et al. Preventing substance use and disordered eating: Initial outcomes of the ATHENA (Athletes Targeting Healthy Exercise and Nutrition Alternatives) program. Arch Pediatr Adolesc Med. 2004;158(11):1043-9.

101. Burns RD, Schiller MR, Merrick MA, et al. Intercollegiate student athlete use of nutritional supplements and the role of athletic trainers and dietitians in nutrition counseling. J Am Diet Assoc. 2004;104(2):246-9.

102. Maughan RJ, Depiesse F, Geyer H. The use of dietary supplements by athletes. J Sports Sci. 2007;25(S1):S103-13.

103. Hoffman JR, Faigenbaum AD, Ratamess NA, et al. Nutritional supplementation and anabolic steroid use in adolescents. Med Sci Sports Exerc. 2008;40(1):15-24.

104. Tscholl P, Alonso JM, Dollé G, et al. The use of drugs and nutritional supplements in top-level track and field athletes. Am J Sports Med. 2010;38(1):133-40.

105. Tsorbatzoudis H, Barkoukis V, Lazuras L. Determinants of Doping Intentions in Sport YOUTH. Montreal: World Anti-Doping Agency; 2013.

106. Ajzen I. The theory of planned behaviour: Reactions and reflections. Psychol Health. 2011;26(9):1113-27.

107. Stewart B, Smith ACT. Drug use in sport: Implications for public policy. J Sport Soc Issues. 2008;32(3):278-98.

108. Geyer H, Parr MK, Koehler K, et al. Nutritional supplements cross-contaminated and faked with doping substances. J Mass Spectrom. 2008;43(7):892-902.

109. Donovan RJ, Egger G, Kapernick V, et al. A conceptual framework for achieving 
performance enhancing drug compliance in sport. Sports Med. 2002;32(4):269-84.

110. Strelan P, Boeckmann RJ. A new model for understanding performance-enhancing drug use by elite athletes. J Appl Sport Psychol. 2003;15(2):176-83.

111. Rivis A, Sheeran P. Descriptive norms as an additional predictor in the theory of planned behaviour: A meta-analysis. Curr Psychol. 2003;22(3):218-33.

112. Armitage CJ, Conner M. Efficacy of the theory of planned behaviour: A meta-analytic review. Br J Soc Psychol. 2001;40(4):471-99.

113. McEachan RRC, Conner M, Taylor NJ, et al. Prospective prediction of health-related behaviours with the theory of planned behaviour: A meta-analysis. Health Psychol Rev. 2011;5(2):97-144.

114. Simons-Morton BG, Haynie D, Noelcke E. Social influences: The effects of socialization, selection, and social normative processes on health behavior. In: DiClemente RJ, Crosby RA, Kegler M, editors. Emerging Theories in Health Promotion Practice and Research. San Francisco, CA: John Wiley \& Sons; 2009. pp. 65-95.

115. Backhouse SH, Patterson L, McKenna J. Achieving the Olympic ideal: Preventing doping in sport. Perform Enhanc Health. 2012;1(2):83-5.

116. Gardner M, Steinberg L. Peer influence on risk taking, risk preference, and risky decision making in adolescence and adulthood: An experimental study. Dev Psychol. 2005;41(4):625-35.

117. Albert D, Chein J, Steinberg L. The teenage brain: Peer influences on adolescent decision making. Curr Dir Psychol Sci. 2013;22(2):114-20.

118. Cialdini RB. Crafting normative messages to protect the environment. Curr Dir Psychol Sci. $2003 ; 12(4): 105-9$.

119. Schultz PW, Nolan JM, Cialdini RB, et al. The constructive, destructive, and reconstructive power of social norms. Psychol Sci. 2007;18(5):429-34. 
Table 1

Results of meta-analyses and homogeneity tests predicting doping behavior and doping intentions

\begin{tabular}{|c|c|c|c|c|c|c|}
\hline & $k$ & $n$ & Effect size & $95 \% \mathrm{CI}$ & FSN & $I^{2}(\%)$ \\
\hline \multicolumn{7}{|l|}{ Staqe one analyses } \\
\hline \multicolumn{7}{|l|}{$\begin{array}{l}\text { Comparisons of intervention and control groups } \\
\text { (Scores of intervention groups minus those of } \\
\text { control groups) }\end{array}$} \\
\hline Behavior (pre- to post-season/school year) & 3 & 3,718 & $0.76^{\mathrm{a}}$ & $0.27,2.17$ & - & 40.8 \\
\hline Behavior (1 year post-intervention) & 1 & 1,291 & $0.36^{\mathrm{a}}$ & $0.12,1.10$ & - & - \\
\hline Intention (pre- to post-season/school year) & 2 & 3,333 & $-0.12^{b}$ & $-0.13,-0.11$ & - & 0.0 \\
\hline \multicolumn{7}{|l|}{ Predicting doping behaviors } \\
\hline \multicolumn{7}{|l|}{ Demographic variables } \\
\hline Sex (males vs. females) & 43 & 247,590 & $2.72^{\mathrm{a}}$ & $2.16,3.42$ & 40 & 84.2 \\
\hline Age & 34 & 182,435 & $-0.01^{\mathrm{c}}$ & $-0.02,0.003$ & - & 82.2 \\
\hline Legal supplement use & 6 & 4,568 & $8.24^{\mathrm{a}}$ & $5.07,13.39$ & 19 & 60.2 \\
\hline Know friend who has doped & 5 & 2,224 & $6.40^{\mathrm{a}}$ & $3.46,11.84$ & 13 & 0.0 \\
\hline \multicolumn{7}{|l|}{ Theory of Planned Behavior variables } \\
\hline Intention ${ }^{\mathrm{d}}$ & 10 & 5,544 & $0.38^{\mathrm{c}}$ & $0.21,0.55$ & 29 & 98.9 \\
\hline Attitudes & 13 & 7,992 & $0.12^{\mathrm{c}}$ & $-0.06,0.30$ & - & 98.7 \\
\hline Knowledge of doping & 1 & 2,285 & $0.08^{\mathrm{c}}$ & $0.04,0.12$ & - & - \\
\hline Perceived benefits of doping & 3 & 1,551 & $0.27^{\mathrm{c}}$ & $0.16,0.37$ & 5 & 82.2 \\
\hline Perceived negative outcomes of doping & 1 & 167 & $-0.08^{\mathrm{c}}$ & $-0.23,0.07$ & - & - \\
\hline
\end{tabular}


Beliefs regarding whether doping should be legalized

Subjective norms

Descriptive norms

Moral norms

Perceived behavioral control

Self-efficacy to refrain from doping

Situational temptations

Other personal psychological variables

Sportspersonship

Moral disengagement

Dissatisfaction with appearance or body image

Autonomous motivation ${ }^{\mathrm{e}}$

Controlled motivation

Amotivation

Task goal orientation ${ }^{\mathrm{e}}$

Ego goal orientation

Global self-esteem

Perfectionism

Religiousness $^{\mathrm{e}}$

Sport confidence

Anticipated regret ${ }^{\mathrm{e}}$

Threat of being caught

Willingness to dope ${ }^{\mathrm{d}}$

\section{Predicting doping intentions}

Demographic variables

Sex (males versus females)

Age

Legal supplement use

Social-contextual environment

Team sports participation (versus other types)

$\begin{array}{rr}205 & 0.52^{\mathrm{c}} \\ 4,084 & 0.36^{\mathrm{c}} \\ 1,233 & 0.49^{\mathrm{c}} \\ 640 & 0.40^{\mathrm{c}} \\ 3,073 & -0.01^{\mathrm{c}} \\ 762 & -0.22^{\mathrm{c}} \\ 949 & 0.47^{\mathrm{c}}\end{array}$

$-0.15^{\mathrm{c}}$

$0.30^{\mathrm{c}}$

$0.15^{\mathrm{c}}$

$-0.06^{\mathrm{C}}$

$0.02^{\mathrm{c}}$

$0.17^{\mathrm{c}}$

$-0.09^{\mathrm{c}}$

$0.04^{\mathrm{c}}$

$-0.03^{\mathrm{c}}$

$-0.10^{\mathrm{c}}$

$-0.58^{\mathrm{C}}$

$-0.04^{\mathrm{c}}$

$-0.13^{\mathrm{c}}$

$0.04^{\mathrm{c}}$

$0.54^{\mathrm{c}}$

$0.42,0.62$

$0.23,0.48$

$0.27,0.70$

$0.33,0.46$

$-0.07,0.05$

$-0.29,-0.16$

$0.42,0.52$

$-0.21,-0.09$

$0.10,0.49$

$0.02,0.29$

$-0.09,-0.03$

$-0.02,0.06$

$0.07,0.26$

$-0.17,-0.01$

$-0.02,0.11$

$-0.07,0.01$

$-0.19,0.001$

$-0.84,-0.32$

$-0.15,0.06$

$-0.21,-0.05$

$-0.04,0.12$

$0.49,0.59$

$0.07^{\mathrm{c}}$

$0.05^{\mathrm{c}}$

6,350

$0.36^{\mathrm{c}}$

$-0.004,0.15$

$0.02,0.09$

$0.20,0.52$

-

90.6

2,110

$0.08^{\mathrm{c}}$

$-0.05,0.21$

95.6

95.4

59.4

$$
34.7
$$

1218

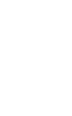


Theory of Planned Behavior variables

Attitudes

Knowledge of doping

Perceived benefits of doping

Perceived negative outcomes of doping

Beliefs regarding whether doping should be legalized

Subjective norms

Descriptive norms

Moral norms

Social support to use illegal substances

Perceived behavioral control

Self-efficacy to refrain from doping

Situational temptations

$\begin{array}{rr}6,878 & 0.55^{\mathrm{c}} \\ 144 & 0.28^{\mathrm{c}} \\ 909 & 0.17^{\mathrm{c}} \\ 421 & -0.25^{\mathrm{c}} \\ 203 & 0.75^{\mathrm{c}} \\ 5,409 & 0.55^{\mathrm{c}} \\ 1,166 & 0.21^{\mathrm{c}} \\ 646 & 0.65^{\mathrm{c}} \\ 144 & 0.33^{\mathrm{c}} \\ 4,456 & -0.08^{\mathrm{c}} \\ 2,102 & -0.55^{\mathrm{c}} \\ 955 & 0.68^{\mathrm{c}}\end{array}$

$0.47,0.63$

$0.13,0.43$

$-0.01,0.35$

$-0.33,-0.16$

$0.69,0.81$

$0.44,0.65$

$0.10,0.32$

$0.60,0.69$

$0.18,0.48$

$-0.27,0.10$

$-0.60,-0.49$

$0.43,0.92$

93.1

Personal psychological variables

Sportspersonship

Moral disengagement

Dissatisfaction with appearance or body image

Drive for thinness

Drive for muscularity

Autonomous motivation

Controlled motivation

Amotivation $^{\mathrm{d}}$

Task goal orientation ${ }^{\mathrm{e}}$

Ego goal orientation ${ }^{\mathrm{d}}$

Global self-esteem

Anticipated regret ${ }^{\mathrm{e}}$

Willingness to dope ${ }^{\mathrm{d}}$

$\begin{array}{rr}1,963 & -0.10^{\mathrm{c}} \\ 2,657 & 0.48^{\mathrm{c}} \\ 529 & 0.19^{\mathrm{c}} \\ 864 & 0.16^{\mathrm{c}} \\ 864 & 0.21^{\mathrm{c}} \\ 2,585 & -0.11^{\mathrm{c}} \\ 2,583 & 0.02^{\mathrm{c}} \\ 2,581 & 0.24^{\mathrm{c}} \\ 2,179 & -0.08^{\mathrm{c}} \\ 2,172 & 0.14^{\mathrm{c}} \\ 191 & -0.03^{\mathrm{c}} \\ 644 & -0.53 \\ 726 & 0.16\end{array}$

$-0.22,0.02$

$0.40,0.57$

$0.03,0.36$

$0.10,0.23$

$0.15,0.28$

$-0.23,0.002$

$-0.04,0.07$

$0.20,0.27$

$-0.14,-0.02$

$0.09,0.20$

$-0.18,0.11$

$-0.59,-0.48$

$0.06,0.24$

\begin{tabular}{cr}
63 & 93.1 \\
2 & - \\
\hline 3 & 93.8 \\
7 & 9.3 \\
49 & - \\
4 & 93.2 \\
6 & -7.7 \\
3 & - \\
-18 & 98.4 \\
12 & 53.9 \\
& 97.8 \\
1 & 89.7 \\
12 & 84.1 \\
3 & 77.8 \\
1 & - \\
2 & - \\
1 & 90.2 \\
\hline 7 & 43.7 \\
\hline 7 & 0.0 \\
2 & 44.3 \\
\hline 5 & -6 \\
1 & - \\
& -
\end{tabular}

\section{Stage two analyses}

Predicting doping behaviors 
Theory of Planned Behavior variables

Attitudes ${ }^{\mathrm{d}}$

16

8,227

$0.17^{\mathrm{c}}$

$0.04,0.29$

11

96.8

(including: knowledge of doping, perceived

benefits of doping, perceived negative outcomes of

doping [reversed], beliefs regarding whether doping should be legalized)

Norms $^{\mathrm{d}}$

(including: subjective norms, descriptive norms, moral norms)

Self-efficacy to refrain from doping ${ }^{\mathrm{e}}$

(including: perceived behavioral control, situational temptations [reversed])

\section{Other personal psychological variables}

Morality ${ }^{\mathrm{e}}$

(including: sportspersonship, moral disengagement [reversed])

\section{Predicting doping intentions}

\section{Theory of Planned Behavior variables}

Attitudes ${ }^{\mathrm{d}}$

(including: knowledge of doping, perceived

benefits of doping, perceived negative outcomes of doping [reversed], beliefs regarding whether doping should be legalized)

Norms $^{\mathrm{d}}$

moral norms, social support to use illegal substances)

Self-efficacy to refrain from doping ${ }^{\mathrm{e}}$ 
Personal psychological variables

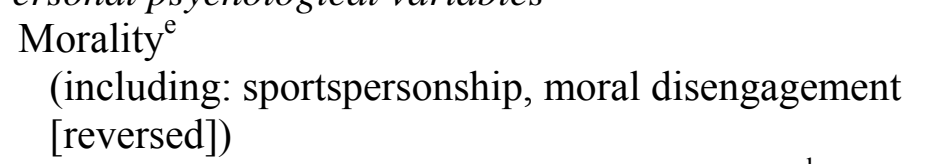

Dissatisfaction with appearance or body image ${ }^{\mathrm{d}}$

(including: drive for thinness, drive for muscularity)

(1)

4,620

$-0.31^{\mathrm{c}}$

$-0.47,-0.16$

13

96.8

$\begin{array}{llllll}4 & 1,393 & 0.20^{\mathrm{c}} & 0.12,0.29 & 5 & 66.6\end{array}$

\section{Stage three analyses}

\section{Predicting doping behaviors}

Positive correlates

Negative correlates

$\begin{array}{rrrccc}17 & 9,297 & 0.20^{\mathrm{c}} & 0.08,0.32 & 17 & 97.2 \\ 11 & 6,538 & -0.13^{\mathrm{c}} & -0.19,-0.07 & 4 & 82.1 \\ & & & & & \\ 15 & 7,875 & 0.44^{\mathrm{c}} & 0.36,0.51 & 51 & 92.5 \\ 11 & 5,819 & -0.28^{\mathrm{c}} & -0.39,-0.17 & 20 & 94.8\end{array}$

\section{Predicting doping intentions}

Positive correlates

Negative correlates

$0.36,0.51$

94.8

Note. $k=$ number of meta-analyzed studies; $n=$ total number of participants; $\mathrm{CI}=$ confidence interval; FSN $=$ fail-safe number, i.e., number of studies with null findings which, if included in the meta-analysis, would reduce the estimated effect to a small size (if it is not already small); -

$=$ not applicable; $I^{2}=$ test of homogeneity (shown when $\mathrm{k}>1$ ). ${ }^{\mathrm{a}}$ Odds ratios; ${ }^{\mathrm{b}} \mathrm{Cohen}$ 's $d ;{ }^{\mathrm{c}}$ Correlation coefficient. ${ }^{\mathrm{d}}$ Variable considered as a positive correlate in stage three analyses; ${ }^{\mathrm{e}}$ Variable considered as an negative correlate in stage three analyses. 
Table 2

Differences in effect size across levels of moderators

\begin{tabular}{|c|c|c|c|c|c|}
\hline Relationship [levels of moderator(s)] & $k$ & $n$ & Effect size & $95 \% \mathrm{CI}$ & $I^{2}(\%)$ \\
\hline \multicolumn{6}{|l|}{ Stage one analyses } \\
\hline \multicolumn{6}{|l|}{ Sex - Doping behaviors ${ }^{\mathrm{a}}$} \\
\hline \multicolumn{6}{|l|}{ Participant background } \\
\hline Competitive athletes & 12 & 4,834 & 2.17 & $1.16,4.08$ & 56.1 \\
\hline Gym users & 4 & 2,692 & 7.77 & $5.31,11.37$ & 36.5 \\
\hline Students & 27 & 240,064 & 2.48 & $2.04,3.00$ & 86.1 \\
\hline \multicolumn{6}{|l|}{ Age - Doping behaviors ${ }^{\mathrm{b}}$} \\
\hline \multicolumn{6}{|l|}{ Sex of participants } \\
\hline Males & 33 & 89,680 & 0.005 & $-0.01,0.02$ & 72.3 \\
\hline Females & 28 & 91,488 & -0.03 & $-0.05,-0.02$ & 73.1 \\
\hline \multicolumn{6}{|l|}{ Study design } \\
\hline Cross-sectional studies & 33 & 181,673 & -0.01 & $-0.02,0.001$ & 77.8 \\
\hline Longitudinal studies & 1 & 762 & 0.22 & $0.15,0.28$ & - \\
\hline \multicolumn{6}{|l|}{ Participant background } \\
\hline Gym users & 5 & 713 & 0.09 & $0.01,0.17$ & 39.0 \\
\hline Students & 18 & 176,377 & -0.01 & $-0.02,0.003$ & 88.5 \\
\hline \multicolumn{6}{|l|}{ Participant age group } \\
\hline Adolescents & 15 & 168,668 & -0.01 & $-0.02,-0.001$ & 81.8 \\
\hline Mixed adults and adolescents & 7 & 6,867 & 0.07 & $0.02,0.13$ & 85.1 \\
\hline \multicolumn{6}{|l|}{ Risk of bias } \\
\hline Studies with low risk of bias & 31 & 179,895 & -0.01 & $-0.02,-0.001$ & 74.8 \\
\hline Studies with potential risk of bias & 3 & 2,540 & 0.14 & $0.07,0.21$ & 78.5 \\
\hline \multicolumn{6}{|l|}{ Intention - Doping behaviors $^{\mathrm{b}}$} \\
\hline \multicolumn{6}{|l|}{ Sport type } \\
\hline Team sports participants & 1 & 236 & -0.07 & $-0.20,0.06$ & - \\
\hline Mixed team and individual sports participants & 7 & 3,946 & 0.44 & $0.25,0.63$ & 98.8 \\
\hline
\end{tabular}




\section{Risk of bias}

Studies with low risk of bias

Studies with potential risk of bias

$9 \quad 5,308 \quad 0.40$

0.40

$0.22,0.57$

$-0.06$

98.9

Attitudes - Doping behaviors ${ }^{\mathrm{b}}$

Risk of bias

Studies with low risk of bias

Studies with potential risk of bias

$11 \quad 5,477$

$2 \quad 2,515$

0.29

$-0.36$

$0.16,0.42$

$-0.48,-0.23$

96.5

Perceived positive effects - Doping behaviors ${ }^{\mathrm{b}}$

Participant age group

Adults

Mixed adults and adolescents

Subjective norms - Doping behaviors ${ }^{\mathrm{b}}$

Sport type

Team sports participants

Mixed team and individual sports participants

Studies with low risk of bias

Studies with potential risk of bias

Descriptive norms - Doping behaviors ${ }^{b}$

\section{Sex of participants}

Males

Females

Participant age group

Adults

Adolescents

Perceived behavioral control - Doping behaviors ${ }^{\mathrm{b}}$

Study design

Cross-sectional studies

Longitudinal studies

Moral disengagement - Doping behaviors ${ }^{\mathrm{b}}$ 
Participant background

Competitive athletes

Students

Amotivation - Doping behaviors ${ }^{\mathrm{b}}$

Participant age group

Adults

Adolescents

Mixed adults and adolescents

Task achievement goal orientation - Doping behaviors ${ }^{\mathrm{b}}$

Participant background

Competitive athletes

Students

Age - Doping intentions ${ }^{\mathrm{b}}$

Study design

Cross-sectional studies

Longitudinal studies

Attitudes - Doping intentions ${ }^{\mathrm{b}}$

Sport type

Team sports participants

Mixed team and individual sports participants

Participant age group

Adults

Mixed adults and adolescents

Perceived benefits - Doping intentions ${ }^{\mathrm{b}}$

Publication status / Study design

Published datasets / Longitudinal studies

Unpublished datasets / Cross-sectional studies

Descriptive norms - Doping intentions ${ }^{\mathrm{b}}$

Participant age group

Adolescents
$1 \quad 644$

$2 \quad 1,714$

0.01

0.40

$-0.06,0.09$

$0.38,0.42$

0.0

$-0.07$

0.20

0.22

$-0.16,0.03$

$0.10,0.31$

$0.17,0.26$

26.8

1,860

$-0.07$

$-0.32$

$-0.13,-0.004$

$-0.44,-0.19$

60.3

$\begin{array}{rr}4 & 2,343 \\ 1 & 200\end{array}$

-

$9 \quad 4,645$

0.07

0.003

$0.04,0.11$

28.0

$2 \quad 1,705$

$0.002,0.004$

0.0

$1 \quad 240$

0.73

0.54

$0.67,0.79$

$0.41,0.66$

94.1

$4 \quad 1,567$

0.38

$0.19,0.56$

$0.63,0.73$

95.8

0.68

70.5

$0.31,0.55$

0.43

$0.03,0.17$

183

0.10

0.07

$-0.04,0.19$ 
Mixed adults and adolescents

Perceived behavioral control - Doping intentions ${ }^{\mathrm{b}}$

\section{Participant background}

Competitive athletes

Gym users

Students

Sport type

Individual sports participants

Mixed team and individual sports participants

Risk of bias

Studies with low risk of bias

Studies with potential risk of bias

Situational temptations - Doping intentions ${ }^{\mathrm{b}}$

Participant age group

Adolescents

Mixed adults and adolescents

Sportspersonship - Doping intentions

Publication status

Published datasets

Unpublished datasets

Moral disengagement - Doping intentions ${ }^{\mathrm{b}}$

Study design / Participant age group

Cross-sectional studies / Mixed adults and adolescents

Longitudinal studies / Adolescents

Dissatisfaction with appearance - Doping intentions ${ }^{\mathrm{b}}$

Participant background

Competitive athletes

Students

Participant age group
1

144

0.40

$0.26,0.53$

$-0.12,0.35$

$-0.93,-0.89$

$-0.22,0.04$

93.1

253

0.11

$-0.09$

$-0.93,-0.89$

$-0.91$

$-0.03$

$-0.19,0.12$

$-0.29,0.09$

$-0.10$

0.35

$0.22,0.47$

0.42

$0.33,0.51$

309

0.80

$0.77,0.83$

$-0.08,0.05$

$-0.31,-0.09$

$2-1,024$

$-0.20$

0.37

$0.31,0.42$

$0.54,0.56$

$2 \quad 1,705$

0.55

0.39

0.06

$0.26,0.51$

$-0.08,0.20$
78.8

0.0

90.5

92.6

98.6

-

-

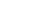


Adults

Mixed adults and adolescents

$1 \quad 182$

0.39

$0.26,0.51$

$2 \quad 347$

0.09

Legal supplement use - Doping intentions ${ }^{\mathrm{b}}$

Participant age group

Adolescents

Mixed adults and adolescents

Autonomous motivation - Doping intentions ${ }^{\mathrm{b}}$

Participant age group

Adults

Adolescents

Mixed adults and adolescents

Controlled motivation - Doping intentions ${ }^{\mathrm{b}}$

Participant background

Competitive athletes

Students

\section{Stage two analyses}

Attitudes (composite) - Doping behaviors ${ }^{\mathrm{b}}$

Risk of bias

Studies with low risk of bias

Studies with potential risk of bias

Norms (composite) - Doping behaviors ${ }^{\mathrm{b}}$

\section{Publication status}

Published datasets

Unpublished datasets

Sport type

Team sports participants

Mixed team and individual sports participants 
Adolescents

Risk of bias

Studies with low risk of bias

Studies with potential risk of bias

Self-efficacy to refrain from doping (composite) -

Doping behaviors ${ }^{\mathrm{b}}$

Participant background

Competitive athletes

Students

Morality (composite) - Doping behaviors ${ }^{\mathrm{b}}$

Study design

Cross-sectional studies

Longitudinal studies

Participant background

Competitive athletes

Students

Attitudes (composite) - Doping intentions ${ }^{\mathrm{b}}$

Sport type

Team sports participants

Individual sports participants

Mixed team and individual sports participants

Participant age group

Adults

Adolescents

Mixed adults and adolescents

Norms (composite) - Doping intentions ${ }^{\mathrm{b}}$

Sport type

Team sports participants

Mixed team and individual sports participants
3

1,299

0.20

$0.06,0.35$

90.3

$8 \quad 3,924$

0.38

$0.29,0.47$

$-0.07,0.19$

65.1

0.06

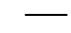

949

$-0.28$

$-0.32,-0.24$

0.0

2,124

$-0.04$

$-0.09,-0.002$

8.6

$6 \quad 4,755 \quad-0.18$

$-0.29,-0.07$

$-0.45,-0.33$

94.5

$-0.19,-0.06$

$-0.42,-0.38$

79.2

$5 \quad 3,803$

$-0.12$

$-0.40$

0.0

$.66,0.78$

$0.47,0.63$

$0.38,0.64$

28.1

0.55

0.51

$0.16,0.45$

0.31

0.54

$0.16,0.45$

$0.59,0.69$

89.0

3,116

0.64

88.0

49.5

$1 \quad 241$

0.70

0.49

$0.64,0.76$

$0.36,0.62$

94.3 
Self-efficacy to refrain from doping (composite) -

Doping intentions ${ }^{\mathrm{b}}$

\section{Participant background}

Competitive athletes

Gym users

Students

Sport type

Individual sports participants

Mixed team and individual sports participants

Morality (composite) - Doping intentions ${ }^{\mathrm{b}}$

Study design

Cross-sectional studies

Longitudinal studies

Participant background

Competitive athletes

Students

$\begin{array}{rrr}3 & 1,137 & -0.32 \\ 2 & 397 & -0.64 \\ 4 & 3,067 & -0.21 \\ & & \\ 2 & 397 & -0.64 \\ 5 & 2,842 & -0.34 \\ & & \\ & & \\ 4 & 2,915 & -0.19 \\ 2 & 1,705 & -0.55 \\ & & \\ 3 & 1,963 & -0.10 \\ 3 & 2,657 & -0.48\end{array}$

$\begin{array}{lr}-0.36,-0.29 & 0.0 \\ -0.88,-0.41 & 91.7 \\ -0.40,-0.02 & 96.0 \\ & \\ -0.88,-0.41 & 91.7 \\ -0.36,-0.31 & 0.0\end{array}$

0.0

96.0

91.7

0.0

Stage three analyses

Positive correlates - Doping behaviors ${ }^{\mathrm{b}}$

Risk of bias

Studies with low risk of bias

Studies with potential risk of bias

Negative correlates - Doping behaviors ${ }^{\mathrm{b}}$

Study design

Cross-sectional studies

Longitudinal studies

Participant background

Competitive athletes

Gym users

Students

$\begin{array}{crr}2 & 2,518 & -0.10 \\ & & \\ 10 & 5,776 & -0.11 \\ 1 & 762 & -0.24 \\ & & \\ 6 & 4,186 & -0.09 \\ 1 & 27 & -0.65 \\ 4 & 2,325 & -0.19\end{array}$

94.8

$-0.13,-0.06 \quad 16.3$

$\begin{array}{ll}-0.22,0.02 & 89.7\end{array}$

$-0.57,-0.40$ 
Sport type

Individual sports participants

1

Mixed team and individual sports participants

Risk of bias

Studies with low risk of bias

Studies with potential risk of bias

$8-5,149$

$10 \quad 6,511$

$1 \quad 27$

Positive correlates - Doping intentions ${ }^{\mathrm{b}}$

Publication status

Published datasets

Unpublished datasets

Study design

Cross-sectional studies

Longitudinal studies

Sport type

Team sports participants

Mixed team and individual sports participants

Negative correlates - Doping intentions ${ }^{\text {b }}$

Study design

Cross-sectional studies

Longitudinal studies

Participant background

Competitive athletes

Gym users

2

397

4,114

$-0.21$

$-0.44$

$-0.16$

$-0.62$

0.49

0.31

$0.40,0.59$

$0.24,0.39$

92.6

0.40

$0.31,0.48$

$0.52,0.61$

92.9

0.56

$0.64,0.77$

$0.29,0.49$

92.6

$0.39 \quad 0.29,0.49$

the moderator. $k=$ number of meta-analyzed studies; $n=$ total number of participants; $\mathrm{CI}=$ confidence interval; $-=$ not applicable; $I^{2}=$ test of homogeneity (shown when $\mathrm{k}>1$ ). ${ }^{\mathrm{a}}$ Odds ratios; ${ }^{\mathrm{b}}$ Correlation coefficient. 


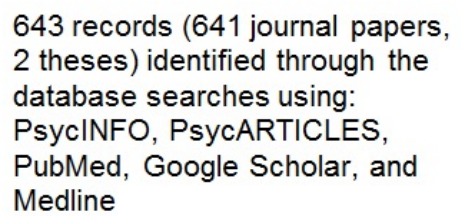

83 records identified through other sources:

61 from authors' existing library;

11 from national database;

7 unpublished datasets;

3 WADA reports;

1 unpublished thesis

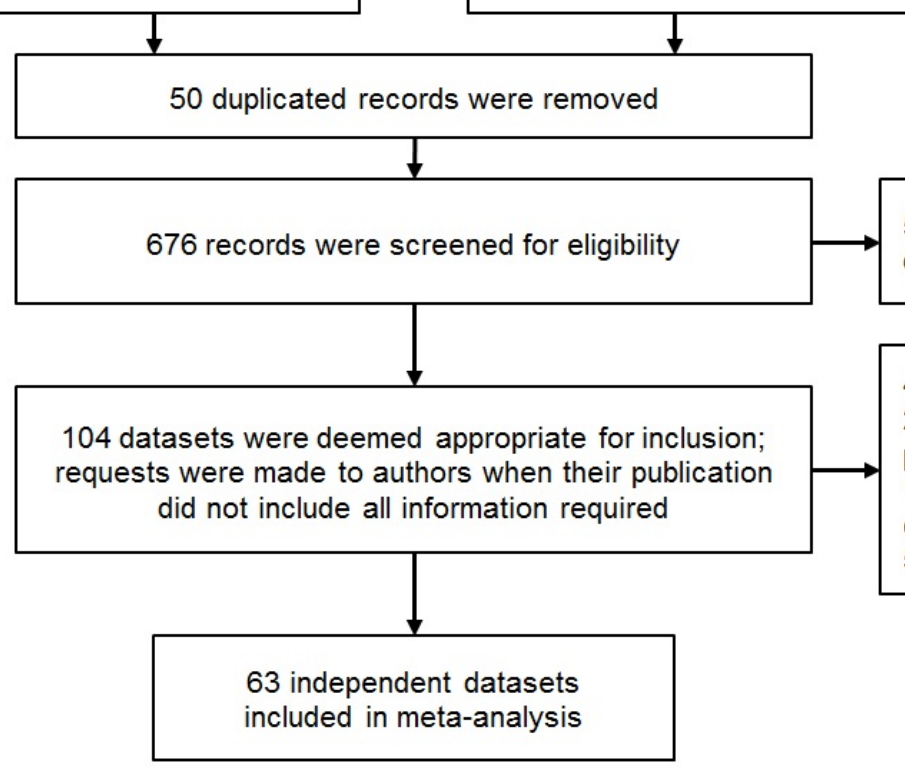

572 records were excluded: did not meet eligibility criteria

41 datasets were excluded: 26 - authors were unable to provide required information; 10 - authors did not respond to our request:

5 - duplicated datasets

Figure 1. PRISMA flow diagram. WADA $=$ World Anti-Doping Agency 


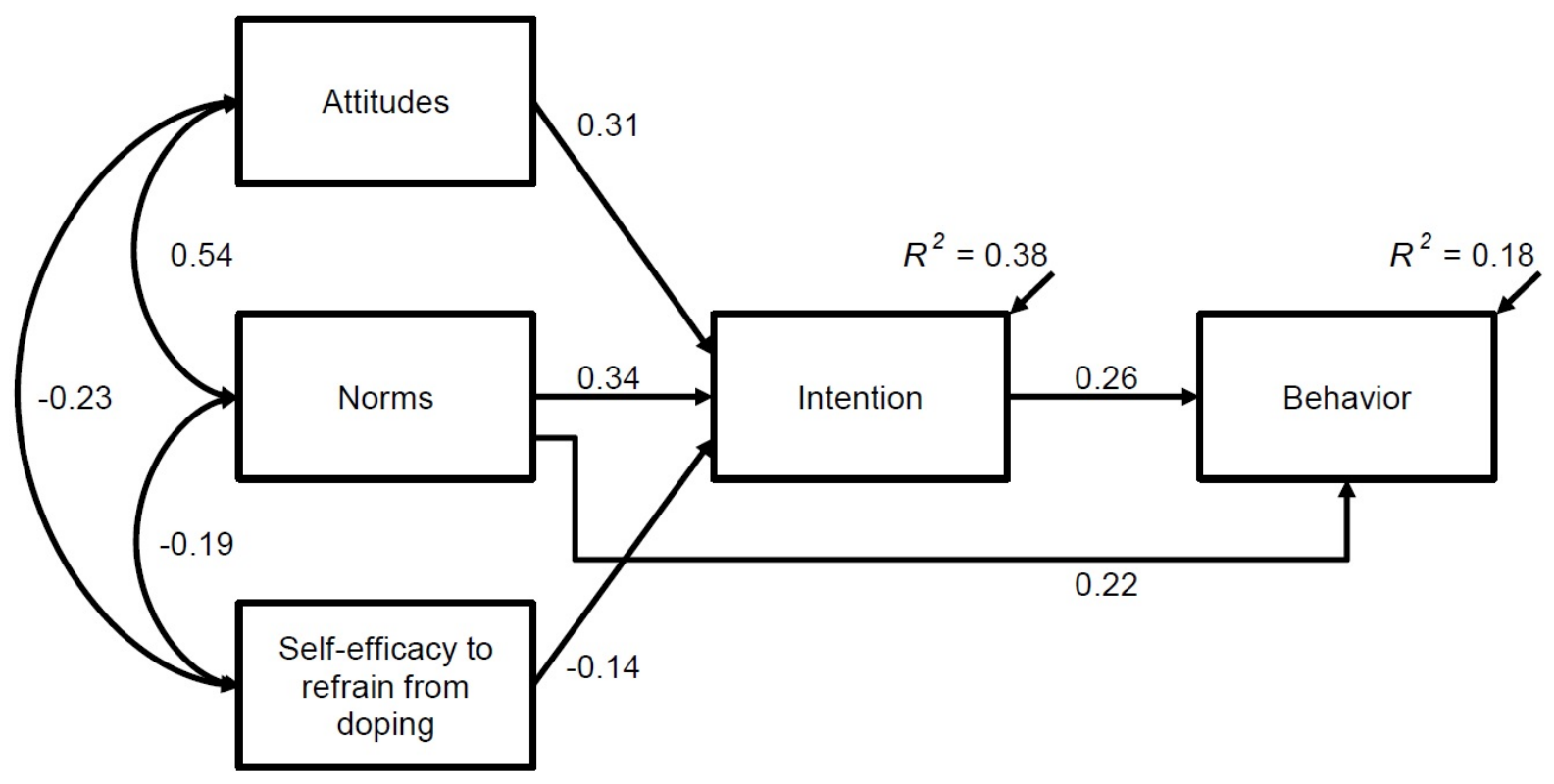

Figure 2. Path diagram of a Theory of Planned Behavior model using meta-analyzed correlations $(k=22, n=5,046)$. All paths are significant at $p<0.001$. 